\title{
Big Bang Nucleosynthesis and Related Observations
}

UMN-TH-1923/00

TPI-MINN-00/47

CERN-TH/2000-287

astro-ph/0009475

Keith A. Olive $e^{1,2, *}$

September 2000

1 TH Division, CERN, Geneva, Switzerland

2 Theoretical Physics Institute, School of Physics and Astronomy, University of Minnesota, Minneapolis MN, USA

* To be published in the Proceedings of Dark 2000: Third International Conference on Dark Matter in Astro and Particle Physics, Heidelberg, Germany, July 10-16 2000

\begin{abstract}
The current status of big bang nucleosynthesis is summarized. Particular attention is paid to recent observations of ${ }^{4} \mathrm{He}$ and ${ }^{7} \mathrm{Li}$ and their systematic uncertainties. Be and $\mathrm{B}$ are also discussed in connection to recent ${ }^{7} \mathrm{Li}$ observations and the primordial ${ }^{7} \mathrm{Li}$ abundance.
\end{abstract}

\section{Introduction}

The simplicity of the standard model of Big Bang Nucleosynthesis (BBN) and its success when confronted with observations place the theory as one of the cornerstones of modern cosmology. BBN is based on the inclusion of an extended nuclear network into a homogeneous and isotropic cosmology. Apart from the input nuclear cross sections, the theory contains only a single parameter, namely the baryon-to-photon ratio, $\eta$. Other factors, such as the uncertainties in reaction rates, and the neutron mean-life can be treated by standard statistical and Monte Carlo techniques 2, 3, 4, 5n. The theory then allows one to make predictions (with specified uncertainties) of the abundances of the light elements, D, ${ }^{3} \mathrm{He},{ }^{4} \mathrm{He}$, and ${ }^{7} \mathrm{Li}$. As there exist several detailed reviews on BBN, I will briefly summarize the key results and devote this contribution to the impact of recent observations of ${ }^{4} \mathrm{He}$ and ${ }^{7} \mathrm{Li}$ along with the related observations of $\mathrm{Be}$ and $\mathrm{B}$. In referring to the standard model, I will mean homogeneous nucleosynthesis, with three neutrino flavors $\left(N_{\nu}=3\right)$, and a neutron mean life of $886.7 \pm 1.9 \mathrm{~s}$ [6].

The dominant product of big bang nucleosynthesis is ${ }^{4} \mathrm{He}$, resulting in an abundance of close to $25 \%$ by mass. Lesser amounts of the other light elements are produced: $\mathrm{D}$ and ${ }^{3} \mathrm{He}$ at the level of about $10^{-5}$ by number, and ${ }^{7} \mathrm{Li}$ at the level of $10^{-10}$ by number. The resulting abundances of the light elements are shown in Figure 1, over the range in $\eta_{10}=10^{10} \eta$ between 1 and 10. The curves for the ${ }^{4} \mathrm{He}$ mass fraction, $Y$, bracket the computed range based mainly on the uncertainty of the neutron mean-life. Uncertainties in the produced ${ }^{7} \mathrm{Li}$ abundances have been adopted from the results in Hata et al.[3]. Uncertainties in $\mathrm{D}$ and ${ }^{3} \mathrm{He}$ production are small on the scale of this figure. The dark shaded boxes correspond to the observed abundances and will be discussed below.

At present, there is a general concordance between the theoretical predictions and the observational data, particularly, for ${ }^{4} \mathrm{He}$ and ${ }^{7} \mathrm{Li}[7]$. These two elements indicate that $\eta$ lies in the range $1.55<\eta<4.45$. There is limited agreement for 


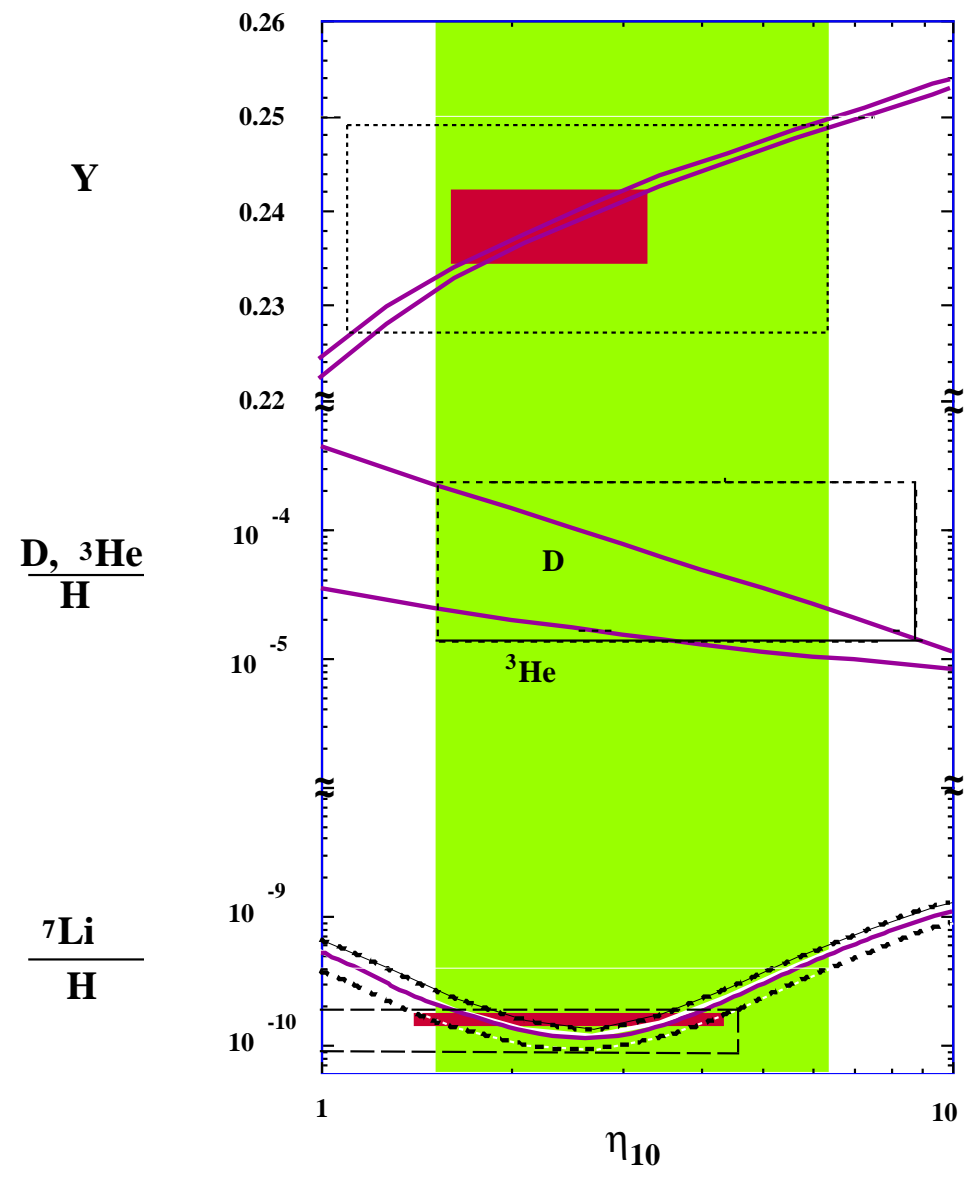

Fig. 1. The light element abundances from big bang nucleosynthesis as a function of $\eta_{10}$.

$\mathrm{D} / \mathrm{H}$ as well, as will be discussed below. High $\mathrm{D} / \mathrm{H}$ narrows the range to $1.5<$ $\eta<3.4$ and low $\mathrm{D} / \mathrm{H}$ is compatible at the $2 \sigma$ level in the range $4.2<\eta<5.3$.

\section{Data}

\section{$2.1{ }^{4} \mathrm{He}$}

The primordial ${ }^{4} \mathrm{He}$ abundance is best determined from observations of HeII $\rightarrow$ HeI recombination lines in extragalactic HII (ionized hydrogen) regions. There is a good collection of abundance information on the ${ }^{4} \mathrm{He}$ mass fraction, $Y, \mathrm{O} / \mathrm{H}$, and $\mathrm{N} / \mathrm{H}$ in over 70 such regions $8,9,10$. Since ${ }^{4} \mathrm{He}$ is produced in stars along with heavier elements such as Oxygen, it is then expected that the primordial 


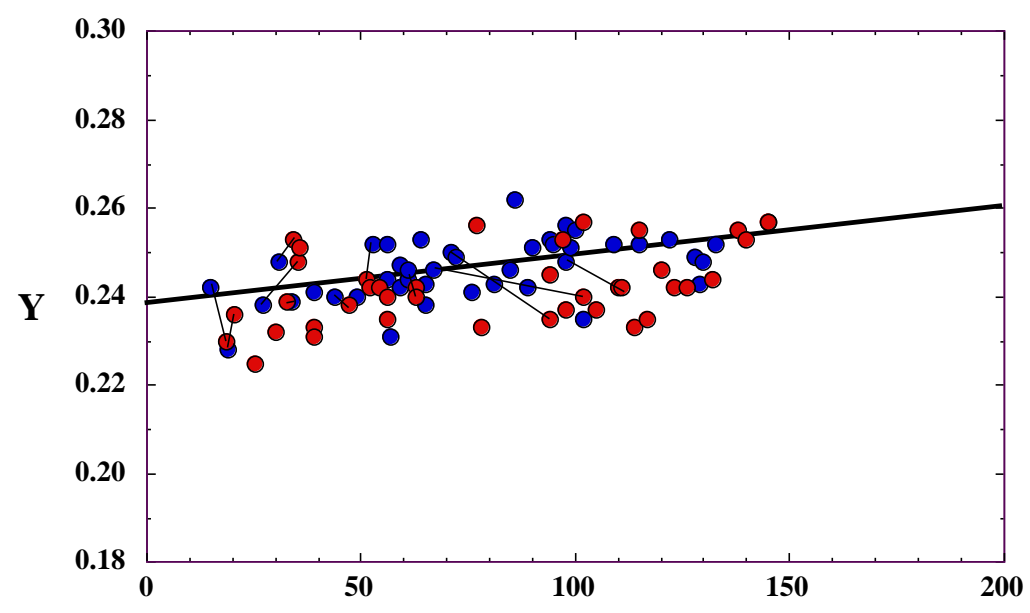

$10^{6} \mathrm{O} / \mathrm{H}$

Fig. 2. The Helium $(\mathrm{Y})$ and Oxygen $(\mathrm{O} / \mathrm{H})$ abundances in extragalactic HII regions, from refs. 8] and from ref. [10. Lines connect the same regions observed by different groups. The regression shown leads to the primordial ${ }^{4} \mathrm{He}$ abundance given in Eq. (1) .

abundance of ${ }^{4} \mathrm{He}$ can be determined from the intercept of the correlation between $Y$ and $\mathrm{O} / \mathrm{H}$, namely $Y_{p}=Y(\mathrm{O} / \mathrm{H} \rightarrow 0)$. A detailed analysis 11 of the data found

$$
Y_{p}=0.238 \pm 0.002 \pm 0.005
$$

The first uncertainty is purely statistical and the second uncertainty is an estimate of the systematic uncertainty in the primordial abundance determination. The solid box for ${ }^{4} \mathrm{He}$ in Figure 1 represents the range (at $2 \sigma_{\text {stat }}$ ) from (1). The dashed box extends this by including the systematic uncertainty. The He data is shown in Figure 2 .

The helium abundance used to derive (1) was determined using assumed electron densities $n$ in the HII regions obtained from SII data. Izotov, Thuan, \& Lipovetsky [9] proposed a method based on several He emission lines to "selfconsistently" determine the electron density. Their data using this method yields a higher primordial value

$$
Y_{p}=0.244 \pm 0.002 \pm 0.005
$$

As one can see, the resulting primordial ${ }^{4} \mathrm{He}$ abundance shows significant sensitivity to the method of abundance determination, leading one to conclude that the systematic uncertainty (which is already dominant) may be underestimated. Indeed, the determination (11) of the primordial abundance above is based on a combination of the data in refs. [8], which alone yield $Y_{p}=0.228 \pm 0.005$, and the data of ref. [10] (based on SII densities) which give $0.239 \pm 0.002$. The abundance (2) is based solely on the self-consistent method yields and the data of 10]. One 
should also note that a recent determination 12 of the ${ }^{4} \mathrm{He}$ abundance in a single object (the SMC) also using the self consistent method gives a primordial abundance of $0.234 \pm 0.003$ (actually, they observe $Y=0.240 \pm 0.002$ at $[\mathrm{O} / \mathrm{H}]$ $=-0.8$, where $[\mathrm{O} / \mathrm{H}]$ refers to the $\log$ of the Oxygen abundance relative to the solar value, in the units used in Figure 2, this corresponds to $10^{6} \mathrm{O} / \mathrm{H}=135$ ). Therefore, it will useful to discuss some of the key sources of the uncertainties in the He abundance determinations and prospects for improvement. To this end, I will briefly discuss, the importance of reddening and underlying absorption in the $\mathrm{H}$ line line measurements, Monte Carlo methods for both $\mathrm{H}$ and $\mathrm{He}$, and underlying absorption in He.

The He abundance is typically quoted relative to $\mathrm{H}$, e.g., He line strengths are measured relative to $H \beta$. The $\mathrm{H}$ data must first be corrected for underlying absorption and reddening. Beginning with an observed line flux $F(\lambda)$, and an equivalent width $W(\lambda)$, we can parameterize the correction for underlying stellar absorption as

$$
X_{A}(\lambda)=F(\lambda)\left(\frac{W(\lambda)+a)}{W(\lambda)}\right)
$$

The parameter $a$ is expected to be relatively insensitive to wavelength. A reddening correction is applied to determine the intrinsic line intensity $I(\lambda)$ relative to $H \beta$

$$
X_{R}(\lambda)=\frac{I(\lambda)}{I(H \beta)}=\frac{X_{A}(\lambda)}{X_{A}(H \beta)} 10^{f(\lambda) C(H \beta)}
$$

where $f(\lambda)$ represents an assumed universal reddening law and $C(H \beta)$ is the correction factor to be determined. By minimizing the differences between $X_{R}(\lambda)$ to theoretical values, $X_{T}(\lambda)$, for $\lambda=H \alpha, H \gamma$ and $H \delta$, one can determine the parameters $a$ and $C(H \beta)$ self consistently [13], and run a Monte Carlo over the input data to test the robustness of the solution and to determine the systematic uncertainty associated with these corrections.

In Figure 3 [13], the result of such a Monte-Carlo based on synthetic data with an assumed correction of $2 \AA$ for underlying absorption and a value for $C(H \beta)=0.1$ is shown. The synthetic data were assumed to have an intrinsic $2 \%$ uncertainty. While the mean value of the Monte-Carlo results very accurately reproduces the input parameters, the spread in the values for $a$ and $C(H \beta)$ are considerably larger than one would have derived from the direct $\chi^{2}$ minimization solution due to the covariance in $a$ and $C(H \beta)$.

The uncertainties found for $H \beta$ must next be propagated into the analysis for ${ }^{4} \mathrm{He}$. We can quantify the contribution to the overall He abundance uncertainty due to the reddening correction by propagating the error in eq. (3). Ignoring all other uncertainties in $X_{R}(\lambda)=I(\lambda) / I(H \beta)$, we would write

$$
\frac{\sigma_{X}}{X}=\ln 10 f(\lambda) \sigma_{C(H \beta)}
$$

In the example discussed above, $\sigma_{C(H \beta)} \sim 0.04$ (from the Monte Carlo), and values of $f$ are $0.237,0.208,0.109,-0.225,-0.345,-0.396$, for He lines at $\lambda \lambda 3889$, $4026,4471,5876,6678,7065$, respectively. For the bluer lines, this correction 


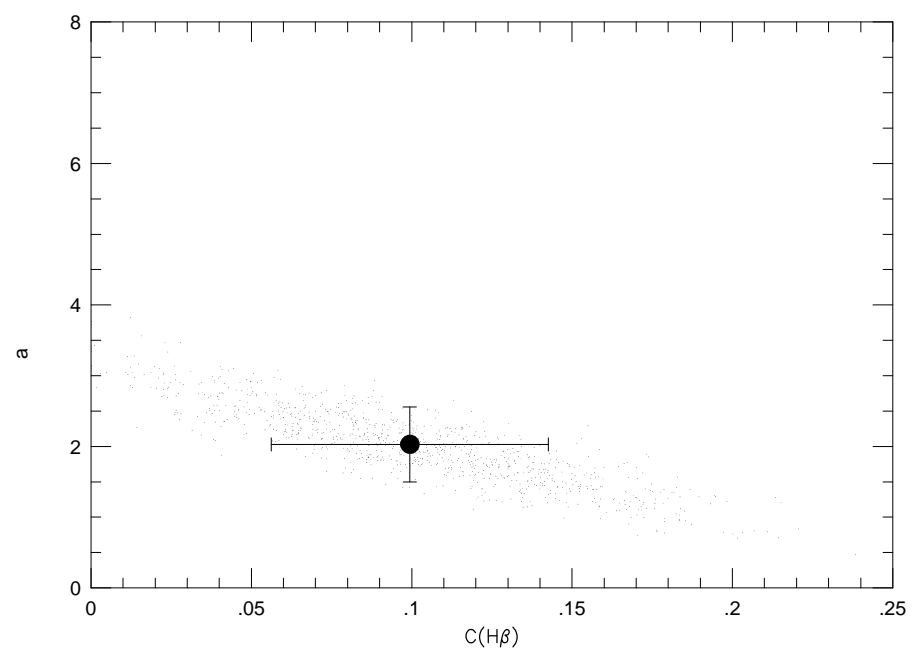

Fig. 3. A Monte Carlo determination of the underlying absorption $a$ (in $\AA$ ), and reddening parameter $C(H \beta)$, based on synthetic data.

alone is $1-2 \%$ and must be added in quadrature to any other observational errors in $X_{R}$. For the redder lines, this uncertainty is $3-4 \%$. This represents the minimum uncertainty which must be included in the individual He I emission line strengths relative to $\mathrm{H} \beta$.

Next one can perform an analogous procedure to that described above to determine the ${ }^{4} \mathrm{He}$ abundance [13]. We again start with a set of observed quantities: line intensities $I(\lambda)$ which include the reddening correction previously determined along with its associated uncertainty which includes the uncertainties in $C(H \beta)$; the equivalent width $W(\lambda)$; and temperature $t$. The Helium line intensities are scaled to $H \beta$ and the singly ionized helium abundance is given by

$$
y^{+}(\lambda)=\frac{I(\lambda)}{I(H \beta)} \frac{E(H \beta)}{E(\lambda)}\left(\frac{W(\lambda)+a_{H e I}}{W(\lambda)}\right) \frac{1}{(1+\gamma)} \frac{1}{f(\tau)}
$$

where $E(\lambda) / E(H \beta)$ is the theoretical emissivity scaled to $H \beta$. The expression (6) also contains a correction factor for underlying stellar absorption, parameterized now by $a_{H e I}$, a density dependent collisional correction factor, $(1+\gamma)^{-1}$, and a flourecence correction which depends on the optical depth $\tau$. Thus $y^{+}$implicitly depends on 3 unknowns, the electron density, $n, a_{H e I}$, and $\tau$.

One can use 3-6 lines to determine the weighted average helium abundance, $\bar{y}$. From $\bar{y}$, we can calculate the $\chi^{2}$ deviation from the average, and minimize $\chi^{2}$, to determine $n, a_{H e I}$, and $\tau$. Uncertainties in the output parameters are also 
determined. In principle, under the assumption of small values for the optical depth $\tau(3889)$, it is possible to use only the three bright lines $\lambda 4471, \lambda 5876$, and $\lambda 6678$ and still solve self-consistently for $\mathrm{He} / \mathrm{H}$, density, and $a_{\mathrm{HeI}}$. Of course, because these lines have relatively low sensitivities to collisional enhancement, the derived uncertainties in density will be large.

The addition of $\lambda 7065$ was proposed [9] as a density diagnostic and then, $\lambda 3889$ was later added to estimate the radiative transfer effects (since these are important for $\lambda 7065$ ). Thus the five line method has the potential of selfconsistently determining the density and optical depth in the addition to the ${ }^{4} \mathrm{He}$ abundance. The procedure described here differs somewhat from that proposed in [9], in that the $\chi^{2}$ above is based on a straight weighted average, where as in [9] the difference of a ratio of He abundances (to one wavelength, say $\lambda 4471$ ) to the theoretical ratio is minimized. When the reference line is particularly sensitive to a systematic effect such as underlying stellar absorption, this uncertainty propagates to all lines this way.

Adding $\lambda 4026$ as a diagnostic line increases the leverage on detecting underlying stellar absorption. This is because the $\lambda 4026$ line is a relatively weak line. However, this also requires that the input spectrum is a very high quality one. $\lambda 4026$ is also provides exceptional leverage to underlying stellar absorption because it is a singlet line and therefore has very low sensitivity to collisional enhancement (i.e., $n$ ) and optical depth (i.e., $\tau(3889)$ ) effects.

As in the case of the hydrogen lines, Monte-Carlo simulation of the He data can be used to test the robustness of the solution for $n, a_{H e I}$, and $\tau$ [13]. Figure 14 presents the results of modeling of 6 synthetic He I line observations. The four panels show the results of a density $=100 \mathrm{~cm}^{-3}, a_{H e I}=0$, and $\tau(3889)=0$ model. The solid lines show the input values (e.g., $\mathrm{He} / \mathrm{H}=0.080$ ) for the original calculated spectrum. The solid circles (with error bars) show the results of the $\chi^{2}$ minimization solution (with calculated errors) for the original synthetic input spectrum. The small points show the results of Monte Carlo realizations of the original input spectrum. The solid squares (with error bars) show the means and dispersions of the output values for the $\chi^{2}$ minimization solutions of the Monte Carlo realizations.

Figure 4 demonstrates several important points. First, the $\chi^{2}$ minimization solution finds the correct input parameters with errors in $\mathrm{He} / \mathrm{H}$ of about $1 \%$ (less than the $2 \%$ errors assumed on the input data, showing the power of using multiple lines). There is a systematic trend for the Monte Carlo realizations to tend toward higher values of $\mathrm{He} / \mathrm{H}$. This is because, the inclusion of errors has allowed minimizations which find lower values of the density and non-zero values of underlying absorption and optical depth. Note that the size of the error bars in $\mathrm{He} / \mathrm{H}$ have expanded by roughly $50 \%$ as a result. We can conclude from this that simply adding additional lines or physical parameters in the minimization does not necessarily lead to the correct results. In order to use the minimization routines effectively, one must understand the role of the interdependencies of the individual lines on the different physical parameters. Here we have shown that trade-offs in underlying absorption and optical depth allow for good solutions at 
Synthetic Data $(T=1.8, E W(H B)=100)$
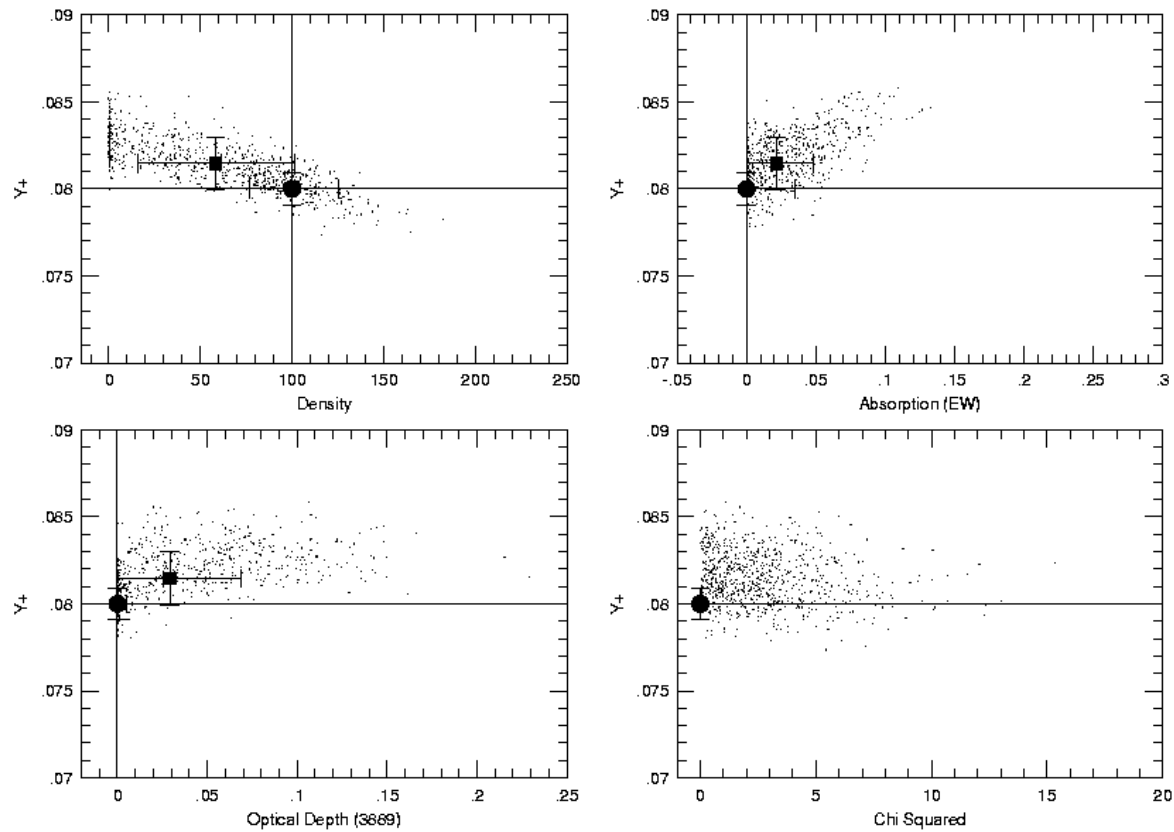

Fig. 4. Results of modeling of 6 synthetic He I line observations. The four panels show the results of a density $=100 \mathrm{~cm}^{-3}, a_{H e I}=0$, and $\tau(3889)=0$ model.

densities which are too low and resulting in helium abundance determinations which are too high. Note that in the lower right panel of Figure 4 that the values of the $\chi^{2}$ do not correlate with the values of $y^{+}$. The solutions at higher values of absorption and $y^{+}$are equally valid as those at lower absorption and $y^{+}$.

Figure 5 shows the results of the Monte Carlo when both $\tau$ and $a_{\mathrm{HeI}} \neq$ 0 , and $n=100 \mathrm{~cm}^{-3}$. It is encouraging that in perhaps more realistic cases where the input parameters are non-zero, we are able to derive results very close to their correct values. The average of Monte Carlo realizations is remarkably close to the straight minimization for all of the derived parameters $\left(n, a_{H e I}, \tau\right.$ and $\left.y^{+}\right)$. However, there is an enormous dispersion in these results due to the degeneracy in the solutions with respect to the physical input parameters. This results in error estimates for parameters which are significantly larger than in the straight minimization. For example, the uncertainties in both the density and optical depth are almost a factor of 3 times larger in the Monte Carlo. When propagated into the uncertainty in the derived value for the He abundance, we find that the uncertainty in the Monte Carlo result (which we argue is a better, not merely more conservative, value) is a factor of 2.5 times the uncertainty obtained from a straight minimization using 6 line He lines. This amounts to an approximately $4 \%$ uncertainty in the He abundance, despite the fact that we assumed (in the synthetic data) $2 \%$ uncertainties in the input line strengths. This 

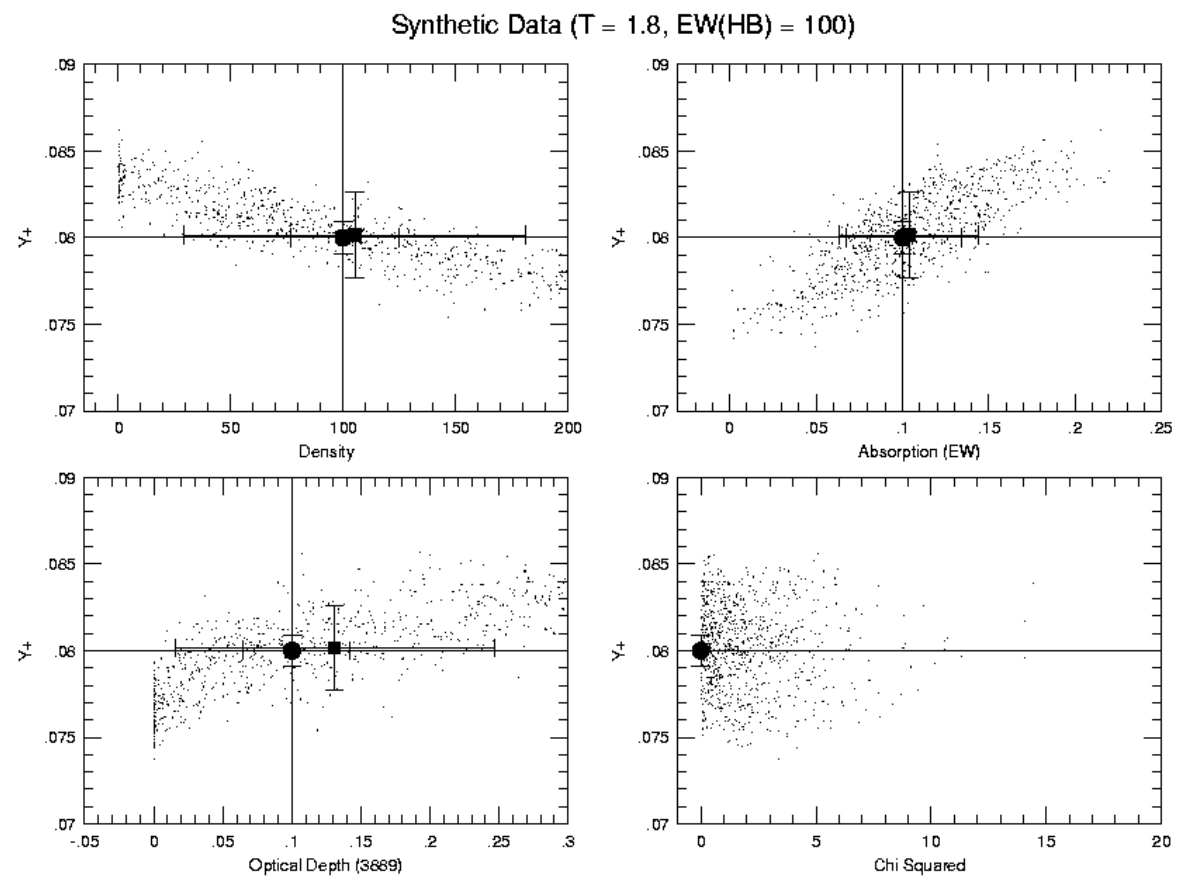

Fig. 5. Similar plot to Figure 4 except that the underlying absorption is $0.1 \AA$ and $\tau(3889)=0.1$.

is an unavoidable consequence of the method - the Monte Carlo routine explores the degeneracies of the solutions and reveals the larger errors that should be associated with the solutions.

In Figure 6, I show the result of a single case based on the data of ref. [10] for SBS1159+545. Here, the helium abundance and density solutions are displayed. The vertical and horizontal lines show the position of the solution in 10]. The circle shows the position of the our solution to the minimization, and the square shows the position of the mean of the Monte-Carlo distribution. The spread shown here is significantly greater than the uncertainty quoted in 10 .

\section{$2.2 \quad{ }^{7} \mathrm{Li}$}

The abundance of ${ }^{7} \mathrm{Li}$ has been determined by observations of over 100 hot, population-II halo stars, and is found to have a very nearly uniform abundance 14. For stars with a surface temperature $T>5500 \mathrm{~K}$ and a metallicity less than about $1 / 20$ th solar (so that effects such as stellar convection may not be important), the abundances show little or no dispersion beyond that which is consistent with the errors of individual measurements. The Li data from Ref. 115] indicate a mean ${ }^{7} \mathrm{Li}$ abundance of

$$
\mathrm{Li} / \mathrm{H}=(1.6 \pm 0.1) \times 10^{-10}
$$




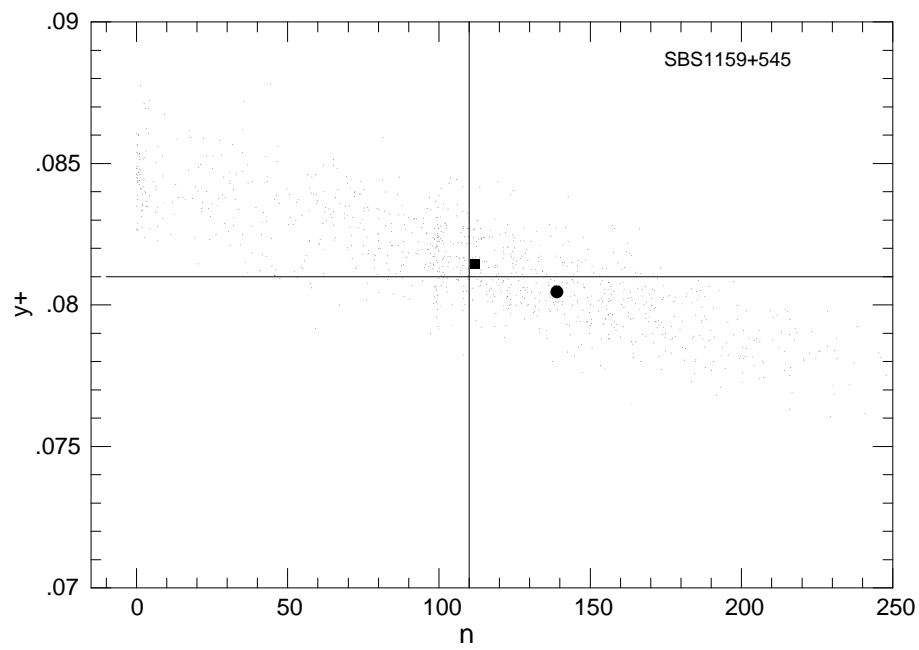

Fig. 6. A Monte Carlo determination of the helium abundance and electron density (in $\mathrm{cm}^{-3}$ ) for the region SBS11159+545. Solutions for $a^{\prime}$ and $\tau$ are not shown here.

The small error is statistical and is due to the large number of stars in which ${ }^{7} \mathrm{Li}$ has been observed. The solid box for ${ }^{7} \mathrm{Li}$ in Figure 11 represents the $2 \sigma_{\text {stat }}$ range from (7).

There is, however, an important source of systematic error due to the possibility that Li has been depleted in these stars, though the lack of dispersion in the Li data limits the amount of depletion. In fact, a small observed slope in $\mathrm{Li}$ vs $\mathrm{Fe}$ and the tiny dispersion about that correlation indicates that depletion is negligible in these stars [16]. Furthermore, the slope may indicate a lower abundance of $\mathrm{Li}$ than that in (6). The observation 17 of the fragile isotope ${ }^{6} \mathrm{Li}$ is another good indication that ${ }^{7} \mathrm{Li}$ has not been destroyed in these stars 18 .

The weighted mean of the ${ }^{7} \mathrm{Li}$ abundance in the sample of ref. [16] is [Li] $=2.12\left([\mathrm{Li}]=\log { }^{7} \mathrm{Li} / \mathrm{H}+12\right)$ and is slightly lower than that in eq. (7) $)$, the difference is a systematic effect due to analysis methods. It is common to test for the presence of a slope in the Li data by fitting a regression of the form $[\mathrm{Li}]=$ $\alpha+\beta[\mathrm{Fe} / \mathrm{H}]$. These data indicate a rather large slope, $\beta=0.07-0.16$ and hence a downward shift in the "primordial" lithium abundance $\Delta[\mathrm{Li}]=-0.20--0.09$. Models of galactic evolution which predict a small slope for $[\mathrm{Li}]$ vs. $[\mathrm{Fe} / \mathrm{H}]$, can produce a value for $\beta$ in the range $0.04-0.07$ [19]. Of course, if we would like to extract the primordial ${ }^{7} \mathrm{Li}$ abundance, we must examine the linear (rather than $\log$ ) regressions. For $\mathrm{Li} / \mathrm{H}=a^{\prime}+b^{\prime} \mathrm{Fe} / \mathrm{Fe}_{\odot}$, we find $a^{\prime}=1-1.2 \times 10^{-10}$ and $b^{\prime}=40-120 \times 10^{-10}$. A similar result is found fitting Li vs O. Overall, when the 
regression based on the data and other systematic effects are taken into account a best value for $\mathrm{Li} / \mathrm{H}$ was found to be 19

$$
\mathrm{Li} / \mathrm{H}=1.23 \times 10^{-10}
$$

with a plausible range between $0.9-1.9 \times 10^{-10}$. The dashed box in Figure 1 corresponds to this range in $\mathrm{Li} / \mathrm{H}$.

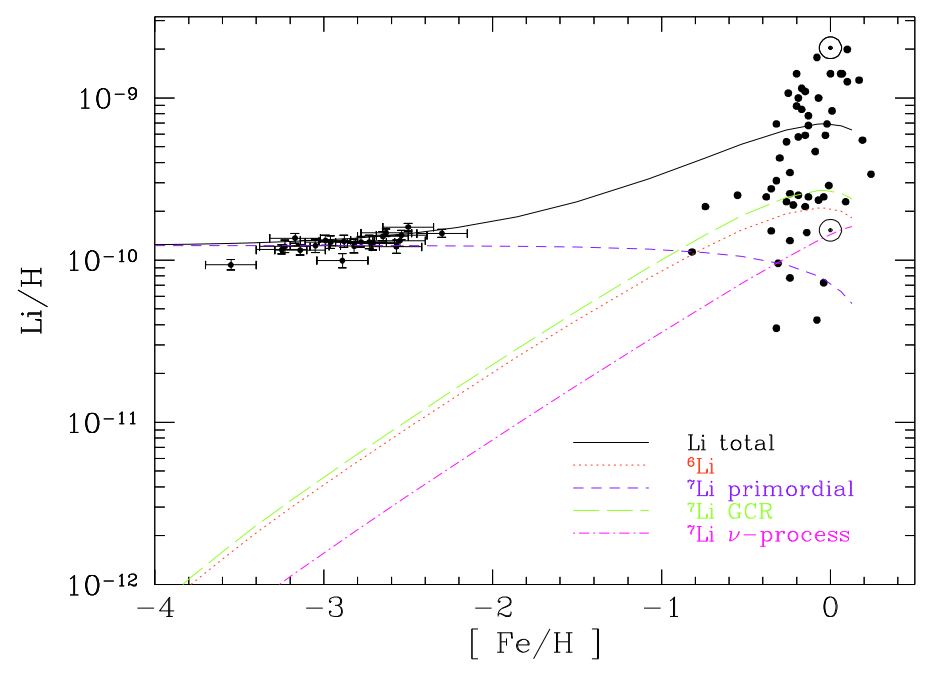

Fig. 7. Contributions to the total predicted lithium abundance from the adopted GCE model of ref. 20], compared with low metallicity stars (from [16]) and a sample of high metallicity stars. The solid curve is the sum of all components.

Figure 7 shows the different Li components for a model with $\left({ }^{7} \mathrm{Li} / \mathrm{H}\right)_{p}=$ $1.23 \times 10^{-10}$. The linear slope produced by the model is $b^{\prime}=65 \times 10^{-10}$, and is independent of the input primordial value (unlike the log slope given above). The model [20] includes in addition to primordial ${ }^{7} \mathrm{Li}$, lithium produced in galactic cosmic ray nucleosynthesis (primarily $\alpha+\alpha$ fusion), and ${ }^{7} \mathrm{Li}$ produced by the $\nu$-process during type II supernovae. As one can see, these processes are not sufficient to reproduce the population I abundance of ${ }^{7} \mathrm{Li}$, and additional production sources are needed.

\section{LiBeB}

The question that one should ask with regard to the above discussion of ${ }^{7} \mathrm{Li}$ (and as we will see below when discussing concordance, ${ }^{7} \mathrm{Li}$ will play an important 
role in determining the baryon density $\eta$ ), is whether or not there is additional evidence for the post big bang production of ${ }^{7} \mathrm{Li}$. There is in fact evidence in the related observation of the intermediate mass elements of ${ }^{6} \mathrm{Li}, \mathrm{Be}$ and $\mathrm{B}$. While these elements are produced in the big bang [21], their predicted primordial abundance is far below their observed abundance, which like ${ }^{7} \mathrm{Li}$ is determined by observations of old metal poor halo stars. Whereas in the range $\eta_{10}=1.5-4.5$, standard BBN predicts abundances of

$$
\begin{aligned}
& { }^{6} \mathrm{Li} / \mathrm{H} \approx(2-9) \times 10^{-14} \\
& { }^{9} \mathrm{Be} / \mathrm{H} \approx(0.04-2) \times 10^{-17} \\
& { }^{10} \mathrm{~B} / \mathrm{H} \approx(0.5-3) \times 10^{-19} \\
& { }^{11} \mathrm{~B} / \mathrm{H} \approx(0.02-1) \times 10^{-16}
\end{aligned}
$$

the observed abundances found in Pop II halo stars are: ${ }^{6} \mathrm{Li} / \mathrm{H} \approx$ few $\times 10^{-12}$, ${ }^{9} \mathrm{Be} / \mathrm{H} \sim 1-10 \times 10^{-13}$, and $\mathrm{B} / \mathrm{H} \sim 1-10 \times 10^{-12}$. It is generally recognized that these isotopes are not of primordial origin, but rather have been produced in the Galaxy, through cosmic-ray nucleosynthesis.

Be and B have been observed in the same pop II stars which show Li and in particular there are a dozen or so stars in which both Be and ${ }^{7} \mathrm{Li}$ have been observed. Thus Be (and B though there is still a paucity of data) can be used as a consistency check on primordial Li. Based on the Be abundance found in these stars, one can conclude that no more than $10-20 \%$ of the ${ }^{7} \mathrm{Li}$ is due to cosmic ray nucleosynthesis leaving the remainder (the abundance in Eq. (8)) as primordial. This is consistent with the conclusion reached in Ref. 19.

In principle, we can use the abundance information on the other LiBeB isotopes to determine the abundance of the associated GCRN produced ${ }^{7} \mathrm{Li}$. As it turns out, the boron data is problematic for this purpose, as there is very likely an additional significant source for ${ }^{11} \mathrm{~B}$, namely $\nu$-process nucleosynthesis in supernovae. Using the subset of the data for which $\mathrm{Li}$ and Be have been observed in the same stars, one can extract the primordial abundance of ${ }^{7} \mathrm{Li}$ in the context of a given model of GCRN. For example, a specific GCRN model, predicts the ratio of $\mathrm{Li} / \mathrm{Be}$ as a function of $[\mathrm{Fe} / \mathrm{H}]$. Under the (plausible) assumption that all of the observed $\mathrm{Be}$ is GCRN produced, the $\mathrm{Li} / \mathrm{Be}$ ratio would yield the GCRN produced ${ }^{7} \mathrm{Li}$ and could then be subtracted from each star to give a set of primordial ${ }^{7} \mathrm{Li}$ abundances. This was done in [22] where it was found that the plateau was indeed lowered by approximately 0.07 dex. However, it should be noted that this procedure is extremely model dependent. The predicted $\mathrm{Li} / \mathrm{Be}$ ratio in GCRN models was studied extensively in [23. It was found that $\mathrm{Li} / \mathrm{Be}$ can vary between 10 and $\sim 300$ depending on the details of the cosmic-ray sources and propagation-e.g., source spectra shapes, escape pathlength magnitude and energy dependence, and kinematics.

In contrast, the ${ }^{7} \mathrm{Li} /{ }^{6} \mathrm{Li}$ ratio is much better determined and far less model dependent since both are predominantly produced by $\alpha-\alpha$ fusion rather than by spallation. The obvious problem however, is the paucity of ${ }^{6} \mathrm{Li}$ data. As more ${ }^{6} \mathrm{Li}$ data becomes available, it should be possible to obtain a better understanding of the relative contribution to ${ }^{7} \mathrm{Li}$ from BBN and GCRN. 
The associated BeB elements are clearly of importance in determining the primordial ${ }^{7} \mathrm{Li}$ abundance, since $\mathrm{Li}$ is produced together with Be and B in accelerated particle interactions such as cosmic ray spallation. However, these production processes are not yet fully understood. Standard cosmic-ray nucleosynthesis is dominated by interactions originating from accelerated protons and $\alpha$ 's on CNO in the ISM, and predicts that BeB should be "secondary" versus the spallation targets, giving $\mathrm{Be} \propto \mathrm{O}^{2}$. However, this simple model was challenged by the observations of BeB abundances in Pop II stars, and particularly the BeB trends versus metallicity. Measurements showed that both Be and B vary roughly linearly with $\mathrm{Fe}$, a so-called "primary" scaling. If O and Fe are co-produced (i.e., if $\mathrm{O} / \mathrm{Fe}$ is constant at low metallicity) then the data clearly contradicts the canonical theory, i.e. BeB production via standard GCR's.

There is growing evidence that the $\mathrm{O} / \mathrm{Fe}$ ratio is not constant at low metallicity 24, but rather increases towards low metallicity. This trend offers a solution to resolve discrepancy between the observed $\mathrm{BeB}$ abundances as a function of metallicity and the predicted secondary trend of GCR spallation [20]. As noted above, standard GCR nucleosynthesis predicts $\mathrm{Be} \propto \mathrm{O}^{2}$, while observations show $\mathrm{Be} \sim \mathrm{Fe}$, roughly; these two trends can be consistent if $\mathrm{O} / \mathrm{Fe}$ is not constant in Pop II. A combination of standard GCR nucleosynthesis, and $\nu$-process production of ${ }^{11} \mathrm{~B}$ may be consistent with current data.

Thus the nature of the production mechanism for BeB (primary vs. secondary) rests with the determination of ratio of $\mathrm{O} / \mathrm{Fe}$ at low metallicity. In any case, it is clear that given a primary mechanism, it will be dominant in the early phases of the Galaxy, and secondary mechanisms will dominate in the latter stage of galactic evolution. The cross over or break point is uncertain. In Figure 8, a plausible model for the evolution of $\mathrm{BeB}$ is shown and compared with the data 25]. Shown by the short dashed lines are standard galactic cosmic-ray nucleosynthesis, which is mostly secondary, but contains some primary production as well. The long dashed curves are purely primary, and in the case of boron, the $\nu$ process has been included and this too is primary. The solid curves represent the total Be and $\mathrm{B}$ abundance as a function of $[\mathrm{O} / \mathrm{H}]$. As one can see such a model fits the data quite well.

\section{Concordance}

Let us now to turn to the question of concordance between the BBN predictions and the observations discussed above. This is best summarized in a comparison of likelihood functions as a function of the one free parameter of BBN, namely the baryon-to-photon ratio $\eta$. By combining the theoretical predictions (and their uncertainties) with the observationally determined abundances discussed above, we can produce individual likelihood functions [7] which are shown in Figure 9 . A range of primordial ${ }^{7} \mathrm{Li}$ values are chosen based on the the abundances in Eqs. (7) and (8) as well as a higher and lower value. The double peaked nature of the ${ }^{7} \mathrm{Li}$ likelihood functions is due to the presence of a minimum in the predicted lithium abundance in the expected range for $\eta$. For a given observed value of 


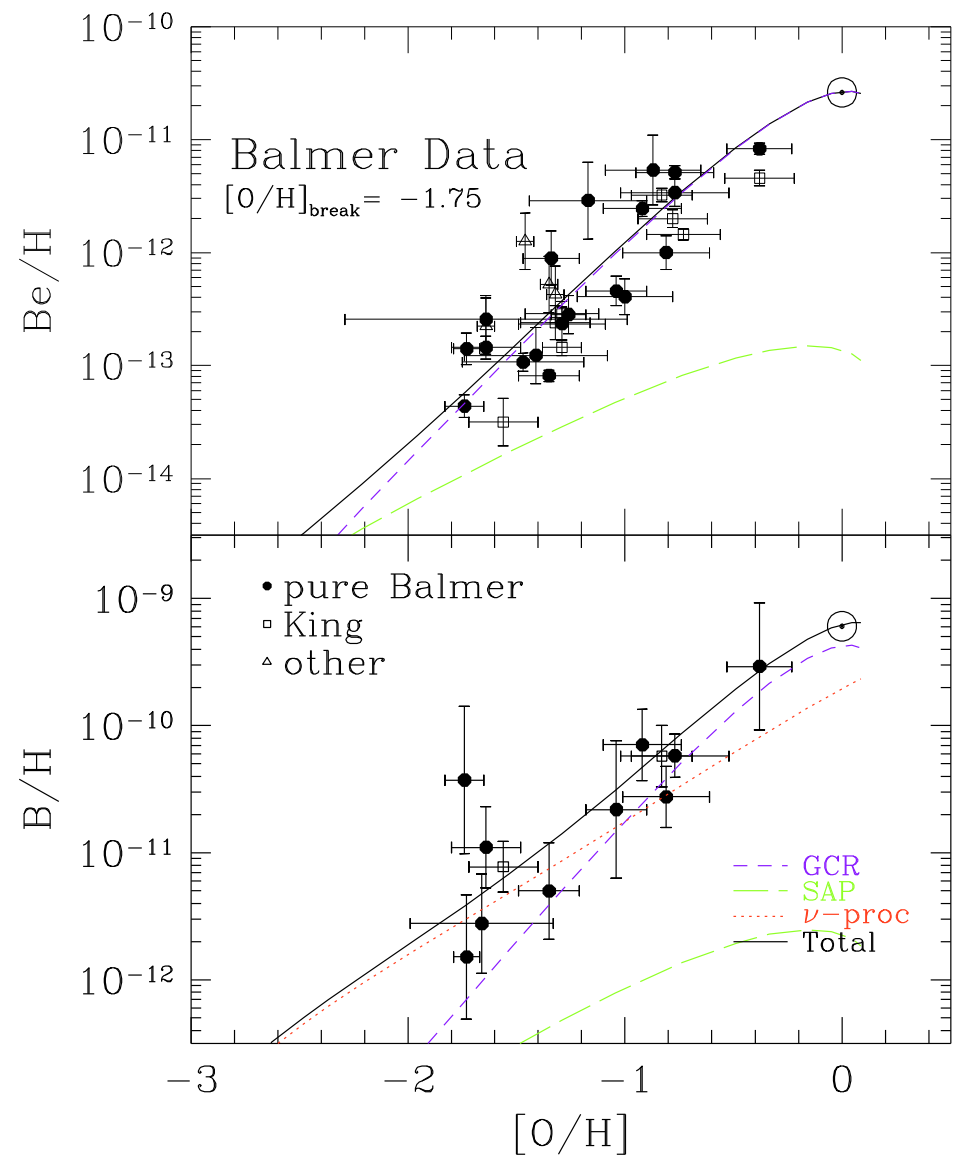

Fig. 8. Be vs $\mathrm{O}$ (top panel) and $\mathrm{B}$ vs $\mathrm{O}$ (bottom panel). Data shown are found to have a break point as indicated. Models are adjusted to achieve the break point and $\mathrm{O} / \mathrm{Fe}$ slope of these data.

${ }^{7} \mathrm{Li}$, there are two likely values of $\eta$. As the lithium abundance is lowered, one tends toward the minimum of the BBN prediction, and the two peaks merge. Also shown are both values of the primordial ${ }^{4} \mathrm{He}$ abundances discussed above. As one can see, at this level there is clearly concordance between ${ }^{4} \mathrm{He},{ }^{7} \mathrm{Li}$ and BBN. 


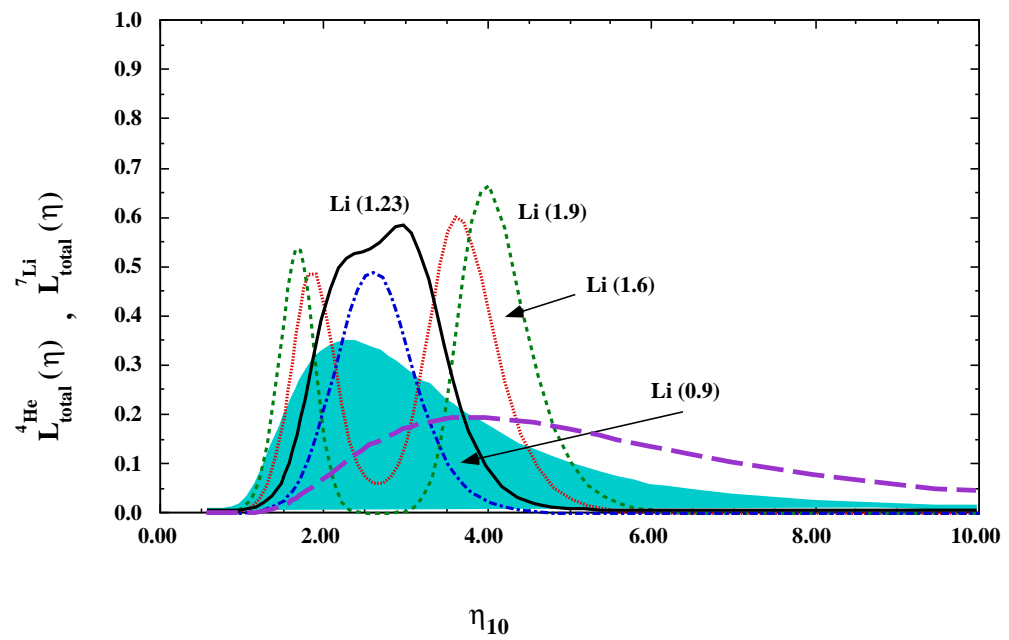

Fig. 9. Likelihood distributions for four values of primordial ${ }^{7} \mathrm{Li} / \mathrm{H}\left(10^{10} \times{ }^{7} \mathrm{Li}=1.9\right.$ (dashed), 1.6 (dotted), 1.23 (solid), and 0.9 (dash-dotted)), and for ${ }^{4} \mathrm{He}$ (shaded) for which we adopt $Y_{p}=0.238 \pm 0.002 \pm 0.005$ (Eq. (1)). Also shown by the long dashed curve is the likelihood function based on the ${ }^{4} \mathrm{He}$ abundance from Eq. (2).

The combined likelihood, for fitting both elements simultaneously, is given by the product of two of the functions in Figure 9 . The combined likelihood is shown in Figure 10, for the two primordial values of ${ }^{7} \mathrm{Li}$ in Eqs. (7) and (8). For ${ }^{7} \mathrm{Li}_{p}=1.6 \times 10^{-10}$ (shown as the dashed curve), the 95\% CL region covers the range $1.55<\eta_{10}<4.45$, with the two peaks occurring at $\eta_{10}=1.9$ and 3.5. This range corresponds to values of $\Omega_{B}$ between

$$
0.006<\Omega_{B} h^{2}<.016
$$

For ${ }^{7} \mathrm{Li}_{p}=1.23 \times 10^{-10}$ (shown as the solid curve), the $95 \%$ CL region covers the range $1.75<\eta_{10}<3.90$. In this case, the primordial value is low enough that the two lithium peaks are more or less merged as is the total likelihood function giving one broad peak centered at $\eta_{10} \simeq 2.5$. The corresponding values of $\Omega_{B}$ in this case are between

$$
0.006<\Omega_{B} h^{2}<.014
$$

When deuterium is folded into the mix, the situation becomes more complicated. Although there are several good measurements of deuterium in quasar absorption systems [26], and many of them giving a low value of $\mathrm{D} / \mathrm{H} \simeq(3.4 \pm$ $0.3) \times 10^{-5}$ [27], there remains an observation with $\mathrm{D} / \mathrm{H}$ nearly an order of magnitude higher $\mathrm{D} / \mathrm{H} \simeq(2.0 \pm 0.5) \times 10^{-4}[28]$.

Because there are no known astrophysical sites for the production of deuterium, all observed D is assumed to be primordial. As a result, any firm determination of a deuterium abundance establishes an upper bound on $\eta$ which is 


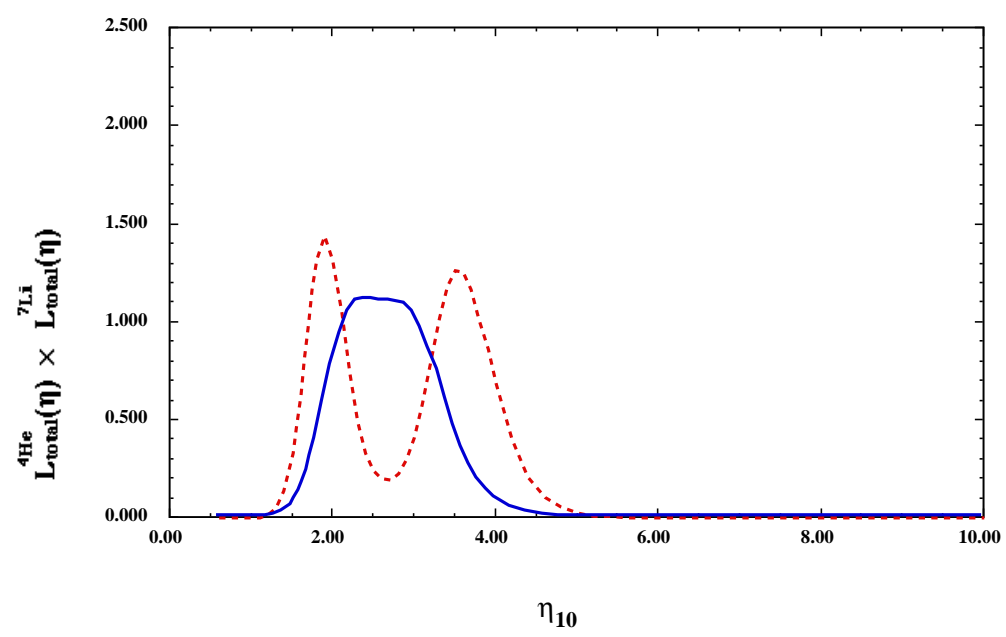

Fig. 10. Combined likelihood distributions for two values of primordial ${ }^{7} \mathrm{Li} / \mathrm{H}\left(10^{10} \times\right.$ ${ }^{7} \mathrm{Li}=1.6$ (dashed), 1.23 (solid)), and ${ }^{4} \mathrm{He}$ with $Y_{p}=0.238 \pm 0.002 \pm 0.005$ (Eq. (1)).

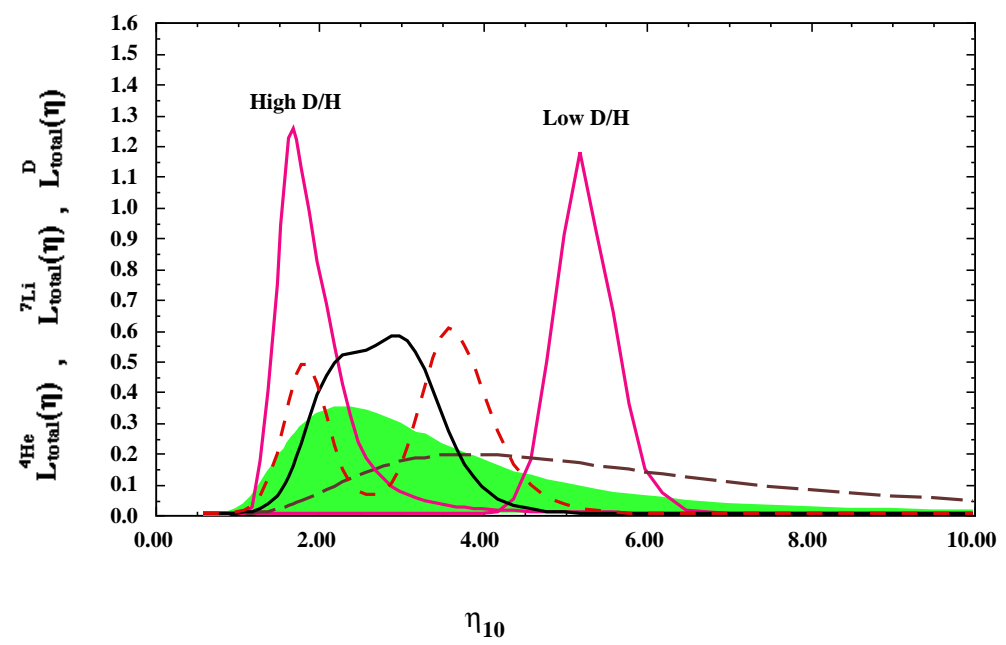

Fig. 11. Likelihood distributions for two values of primordial ${ }^{7} \mathrm{Li} / \mathrm{H}\left(10^{10} \times{ }^{7} \mathrm{Li}=1.6\right.$ (dashed) and 1.23 (solid)), and ${ }^{4} \mathrm{He}$ with $Y_{p}=0.238 \pm 0.002 \pm 0.005$ from Eq. (1) (shaded) and $Y_{p}=0.244 \pm 0.002 \pm 0.005$ from Eq. (2) (long dashed). Also shown are the two likelihood functions for high and low $\mathrm{D} / \mathrm{H}$ as marked.

robust. Thus the ISM measurements 29] of $\mathrm{D} / \mathrm{H}=1.6 \times 10^{-5}$ imply an upper bound $\eta_{10}<9$.

It is interesting to compare the results from the likelihood functions of ${ }^{4} \mathrm{He}$ and ${ }^{7} \mathrm{Li}$ with that of $\mathrm{D} / \mathrm{H}$. This comparison is shown in Figure 11. Using the higher value of $\mathrm{D} / \mathrm{H}=(2.0 \pm 0.5) \times 10^{-4}$, we would find excellent agreement 
between ${ }^{4} \mathrm{He},{ }^{7} \mathrm{Li}$ and $\mathrm{D} / \mathrm{H}$. The predicted range for $\eta$ now becomes

$$
1.6<\eta_{10}<3.2
$$

with the peak likelihood value at $\eta_{10}=2.1,{ }^{4} \mathrm{He}$ and ${ }^{7} \mathrm{Li}$ abundances from eqs. (11) and (8) respectively. This corresponds to $\Omega_{B} h^{2}=.008_{-.002}^{+.004}$. The higher ${ }^{7} \mathrm{Li}$ abundance of eq. (7) drops the peak value down slightly to $\eta_{10}=1.8$ and broadens the range to $1.5-3.4$. The higher ${ }^{4} \mathrm{He}$ abundance shifts the peak and range (relative to eq. (12)) up to 2.2 and $1.7-3.5$.

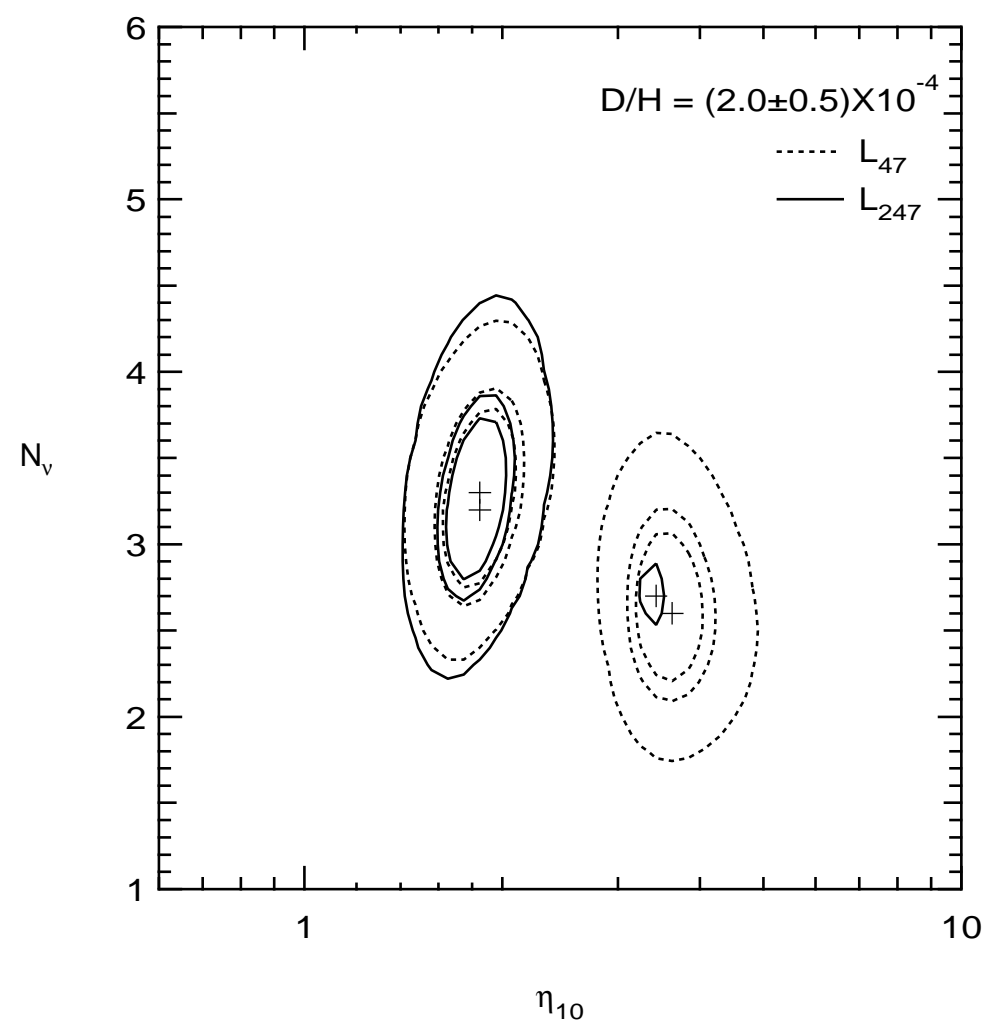

Fig. 12. $50 \%, 68 \%$ \& $95 \%$ C.L. contours of $L_{47}$ and $L_{247}$ where observed abundances are given by eqs. (1 and 7), and high $\mathrm{D} / \mathrm{H}$.

If instead, we assume that the low value of $\mathrm{D} / \mathrm{H}=(3.4 \pm 0.3) \times 10^{-5}[27$ is the primordial abundance, there is hardly any overlap between the $\mathrm{D}$ and ${ }^{7} \mathrm{Li}$, particularly for the lower value of ${ }^{7} \mathrm{Li}$ from eq. (8). There is also very limited overlap between D/H and ${ }^{4} \mathrm{He}$, though because of the flatness of the ${ }^{4} \mathrm{He}$ abundance with respect to $\eta$, as one can see, the likelihood function for the larger value of ${ }^{4} \mathrm{He}$ from eq. (2) is very broad. In this case, $\mathrm{D} / \mathrm{H}$ is just compatible 
(at the $2 \sigma$ level) with the other light elements, and the peak of the likelihood function occurs at roughly $\eta_{10}=4.8$ and with a range of $4.2-5.6$.

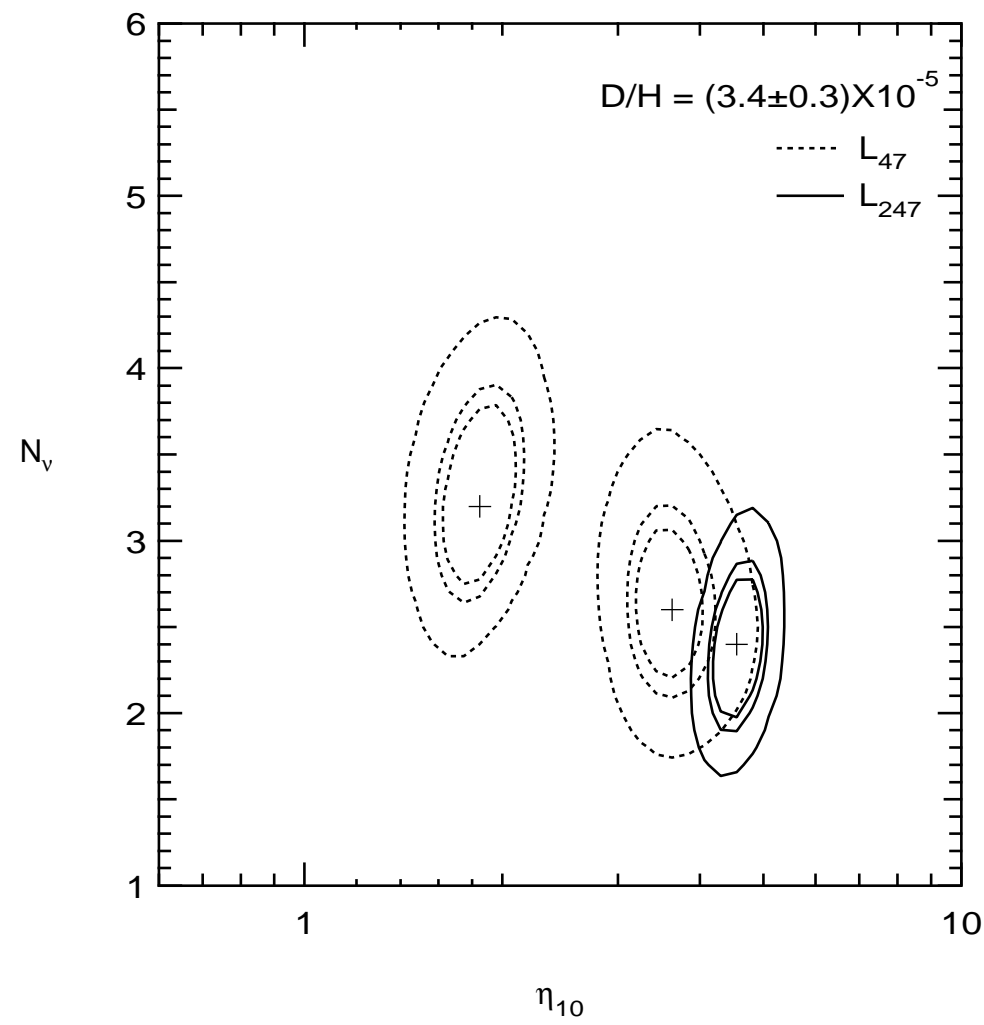

Fig. 13. $50 \%, 68 \% \& 95 \%$ C.L. contours of $L_{47}$ and $L_{247}$ where observed abundances are given by eqs. (11 and 7 ), and low $\mathrm{D} / \mathrm{H}$.

It is important to recall however, that the true uncertainty in the low $\mathrm{D} / \mathrm{H}$ systems might be somewhat larger. Mesoturbulence effects 30] allow $\mathrm{D} / \mathrm{H}$ to be as large as $5 \times 10^{-5}$. In this case, the peak of the $\mathrm{D} / \mathrm{H}$ likelihood function shifts down to $\eta_{10} \simeq 4$, and there would be a near perfect overlap with the high $\eta$ ${ }^{7} \mathrm{Li}$ peak and since the ${ }^{4} \mathrm{He}$ distribution function is very broad, this would be a highly compatible solution.

We can obtain still more information regarding the compatibility of the observed abundance and BBN by considering generalized likelihood functions where we allow $N_{\nu}$ to vary as well [7,31,32, 1 . The likelihood functions now become functions of two parameters $\mathcal{L}\left(\eta, N_{\nu}\right)$.

The peaks of the distribution as well as the allowed ranges of $\eta$ and $N_{\nu}$ are easily discerned in the contour plots of Figures 12 and 13 which show the $50 \%$, $68 \%$ and $95 \%$ confidence level contours in $L_{47}$ and $L_{247}$ projected onto the $\eta$ - 
$N_{\nu}$ plane, for high and low $\mathrm{D} / \mathrm{H}$ as indicated. $L_{47}$ corresponds to the likelihood function based on ${ }^{4} \mathrm{He}$ and ${ }^{7} \mathrm{Li}$ only, whereas $L_{247}$ includes $\mathrm{D} / \mathrm{H}$ as well. The crosses show the location of the peaks of the likelihood functions. $L_{47}$ peaks at $N_{\nu}=3.2, \eta_{10}=1.85$ and at $N_{\nu}=2.6, \eta_{10}=3.6$. The $95 \%$ confidence level allows the following ranges in $\eta$ and $N_{\nu}$

$$
1.7 \leq N_{\nu} \leq 4.3 \quad 1.4 \leq \eta_{10} \leq 4.9
$$

Note however that the ranges in $\eta$ and $N_{\nu}$ are strongly correlated as is evident in Figure 12.

With high $\mathrm{D} / \mathrm{H}, L_{247}$ peaks at $N_{\nu}=3.3$, and also at $\eta_{10}=1.85$. In this case the $95 \%$ contour gives the ranges

$$
2.2 \leq N_{\nu} \leq 4.4 \quad 1.4 \leq \eta_{10} \leq 2.4
$$

Note that within the $95 \%$ CL range, there is also a small area with $\eta_{10}=3.2-3.5$ and $N_{\nu}=2.5-2.9$.

Similarly, for low D/H, $L_{247}$ peaks at $N_{\nu}=2.4$, and $\eta_{10}=4.55$. The $95 \%$ CL upper limit is now $N_{\nu}<3.2$, and the range for $\eta$ is $3.9<\eta_{10}<5.4$. It is

important to stress that these abundances are now consistent with the standard model value of $N_{\nu}=3$ at the $2 \sigma$ level.

\section{Acknowledgments}

This work was supported in part by DoE grant DE-FG02-94ER-40823 at the University of Minnesota.

\section{References}

1. T.P. Walker, G. Steigman, D.N. Schramm, K.A. Olive and K. Kang, Ap.J. 376 (1991) 51; S. Sarkar, Rep. Prog. Phys. 59 (1996) 1493;

K.A. Olive, astro-ph/9901231; K.A. Olive, G. Steigman, and T.P. Walker, Phys. Rep. 333-334 (2000) 389.

2. L.M. Krauss and P. Romanelli, Ap.J. 358 (1990) 47; M. Smith, L. Kawano, and R.A. Malaney, Ap.J. Supp. 85 (1993) 219.

3. N. Hata, R.J. Scherrer, G. Steigman, D. Thomas, and T.P. Walker, Ap.J. 458 (1996) 637.

4. G. Fiorentini, E. Lisi, S. Sarkar, and F.L. Villante, Phys.Rev. D58 (1998) 063506.

5. K. M. Nollett and S. Burles, Phys. Rev. D61 (2000) 123505.

6. D.E. Groom et al. Eur. Phys. J. C15 (2000) 1.

7. B.D. Fields and K.A. Olive, Phys. Lett. B368 (1996) 103;

B.D. Fields, K. Kainulainen, D. Thomas, and K.A. Olive, New Ast. 1 (1996) 77.

8. B.E.J. Pagel, E.A. Simonson, R.J. Terlevich and M. Edmunds, MNRAS 255 (1992) 325 ;

E. Skillman and R.C. Kennicutt, Ap.J. 411 (1993) 655;

E. Skillman, R.J. Terlevich, R.C. Kennicutt, D.R. Garnett, and E. Terlevich, Ap.J. 431 (1994) 172. 
9. Y.I. Izotov, T.X. Thuan, and V.A. Lipovetsky, Ap.J. 435 (1994) 647; Ap.J.S. 108 (1997) 1.

10. Y.I. Izotov, and T.X. Thuan, Ap.J. 500 (1998) 188.

11. K.A. Olive, E. Skillman, and G. Steigman, Ap.J. 483 (1997) 788; B.D. Fields and K.A. Olive, Ap.J. 506 (1998) 177.

12. M. Peimbert, A. Peimbert, and M.T. Ruiz, astro-ph/0003154.

13. K.A. Olive and E. Skillman, astro-ph/0007081.

14. F. Spite, and M. Spite, A.A. 115 (1982) 357.

15. P. Molaro, F. Primas, and P. Bonifacio, A.A. 295 (1995) L47; P. Bonifacio and P. Molaro, MNRAS 285 (1997) 847.

16. S.G. Ryan, J.E. Norris, and T.C. Beers, Ap.J. 523 (1999) 654.

17. V.V. Smith, D.L. Lambert, and P.E. Nissen, Ap.J. 408 (1992) 262; Ap.J. 506 (1998) 405;

L. Hobbs, and J. Thorburn, Ap.J. 428 (1994) L25; Ap.J. 491 (1997) 772;

R. Cayrel, M. Spite, F. Spite, E. Vangioni-Flam, M. Cassé, and J. Audouze, A.A. 343 (1999) 923.

18. G. Steigman, B. Fields, K.A. Olive, D.N. Schramm, and T.P. Walker, Ap.J. 415 (1993) L35;

M. Lemoine, D.N. Schramm, J.W. Truran, and C.J. Copi, Ap.J. 478 (1997) 554;

M.H. Pinsonneault, T.P. Walker, G. Steigman, and V.K. Naranyanan, Ap.J. 527, 180 (1998);

B.D.Fields and K.A. Olive, New Astronomy, 4 (1999) 255;

E. Vangioni-Flam, M. Cassé, R. Cayrel, J. Audouze, M. Spite, and F. Spite, New Astronomy, 4 (1999) 245.

19. S.G. Ryan, T.C. Beers, K.A. Olive, B.D. Fields, and J.E. Norris, Ap.J. Lett. 530 (2000) L57.

20. B. Fields and K.A. Olive, Ap.J. 506, 177 (1998).

21. D. Thomas, D.N. Schramm, K.A. Olive, and B.D. Fields, Ap.J. 406 (1993) 569.

22. K.A. Olive, and D.N. Schramm, Nature 360 (1993) 439.

23. B.D. Fields, K.A. Olive, and D.N. Schramm, Ap.J. 435 (1994) 185.

24. G. Israelian, R.J. García-López, and R. Rebolo, Ap.J. 507, 805 (1998);

A.M. Boesgaard, J.R. King, C.P. Deliyannis, and S.S. Vogt, A.J. 117, 492 (1999).

25. B.D. Fields, K.A. Olive, E. Vangioni-Flam, and M. Cassé, Ap.J. 540 (2000) 930.

26. S. Burles, these proceedings.

27. S. Burles and D. Tytler, Ap.J. 499 (1998) 699; Ap.J. 507 (1998) 732.

28. J.K. Webb, R.F. Carswell, K.M. Lanzetta, R. Ferlet, M. Lemoine, A. Vidal-Madjar, and D.V. Bowen, Nature 388 (1997) 250;

D. Tytler, S. Burles, L. Lu, X.-M. Fan, A. Wolfe, and B.D. Savage, A.J. 117 (1999) 63.

29. J.L. Linsky, et al., Ap.J. 402 (1993) 694; J.L. Linsky, et al.,Ap.J. 451 (1995) 335.

30. S. Levshakov, D. Tytler, and S. Burles, astro-ph/9812114.

31. K.A. Olive and D. Thomas, Astro. Part. Phys. 7 (1997) 27.

32. K.A. Olive and D. Thomas, Astro. Part. Phys. 11 (1999) 403. 